(c) 2002 VSP International Science Publishers. Reprinted with permission from P.A. Belov, S.A. Tretyakov, A.J. Viitanen, Journal of Electromagnetic Waves and Applications, vol. 16, no. 8, pp. 1153-1170, 2002.

J. of Electromagn. Waves and Appl., Vol. 16, No. 8, 1153-1170, 2002

\title{
DISPERSION AND REFLECTION PROPERTIES OF ARTIFICIAL MEDIA FORMED BY REGULAR LATTICES OF IDEALLY CONDUCTING WIRES
}

\author{
P. A. Belov, S. A. Tretyakov, and A. J. Viitanen \\ Radio Laboratory \\ Helsinki University of Technology \\ P.O. Box 3000, FIN-02015 HUT, Finland
}

\begin{abstract}
An analytical theory of electromagnetic waves in artificial media formed by a rectangular lattice of thin ideally conducting cylinders using the local field approach is developed. As a result, the transcendental dispersion equation is obtained in closed form and solved numerically. Typical dispersion curves are calculated. Using these results, the reflection problem from an interface between a half space of wire medium and free space is solved for plane-wave excitation. In the low-frequency approximation a simple analytical formula for the frequency dependent effective dielectric permittivity is established.
\end{abstract}

\section{Introduction}

2 Formulation of the Problem

3 Dispersion Equation

4 Reflection Coefficient

5 Dense Grid

6 Conclusion

\section{References}

\section{INTRODUCTION}

Artificial materials for application in microwave regime have been known for a long time. One of the most attractive features of composite materials is the fact that some unique and exotic material properties can be realized. For example, new realizations and new applications 
for composite media with negative permittivity and permeability were suggested recently. One possible realization of material with negative permittivity is the use of a three-dimensional lattice formed by thin parallel conducting wires. This structure is known in the microwave engineering for a long time as an artificial dielectric [1, 21], and we will call it wire medium. In the literature wire media have received increasing attention recently because of potential new applications, for example as antenna reflectors. They can be used also in the synthesis of artificial impedance surfaces [2]. More recently, wire media formed by long but finite-length wires were studied [3], and some new interesting phenomena described, such as angular windows of wave propagation.

This paper presents the results of an analytical investigation of electromagnetic properties of wire media. The results describe eigenwave properties and plane-wave reflection from an interface with such a material, and give a simple model for the medium parameter in the quasi-static regime.

There are many known numerical techniques and enough complicated theories describing electromagnetic properties of the structure of our interest. For example, in [4] the propagation of electromagnetic waves through arrays (square and hexagonal) of perfectly conducting cylinders for both fundamental polarizations is studied using generalized Rayleigh method (expansion by cylindrical harmonics). The properties of dispersion diagrams are investigated and a comparison with the quasistatic limit is done. The Green functions and lattice sums are calculated by an original method [5].

In the optical frequency band near the plasmon resonance metals can be effectively described by a dielectric permittivity depending on the frequency. In this case in electrodynamic terms a square grid of metallic cylinders is modelled as an equivalent grid of dielectric cylinders with a frequency dependent permittivity. The dispersion characteristics of such metallic systems are studied in [6-9] using the plane-wave expansion method, and the transmission characteristics are considered in [10, 11] using the transfer matrix method [12]. All these methods can be successfully used for perfectly conducting cylinders also.

A lot of attention is paid to discussions of a low-frequency band gap existence in [13]. The analysis is based on calculations using computer codes based on rigorous theories of scattering. The authors show that the structure can be simulated as a material that has a plasmon frequency in the microwave domain and can be used as an antenna substrate.

A very interesting approach to the analysis of media formed by long but finite wires is presented in [3]. The material is split into its 
elementary planes, and the periodic method of moments is applied for characterization of each plane. The effective wavenumber is determined from the theory of periodic structures. The artificial material has a small propagation band at low frequencies due to the finite size of wires and a classical bandgap at higher frequencies.

Although there are several available numerical techniques for modelling of metallic periodic grids of infinite cylinders, there has been very modest progress in developing exact and approximate analytical models. The well known method based on the transmission line theory [1] is accurate only if the ratio between the lattice constants of the media is sufficiently large (in this case the quasistatic approach can be applied for the description of interaction in densely packed planes of wires and the single-mode approach for the interaction between these planes). The analytical results in [4] are too complex. A considerably simpler analytical method for the calculation of the band structure of grids from ideally conducting cylinders is presented in [14]. That approach is based on ideas of spatial averaging.

Here we introduce a simple and in the same time exact analytical theory of dispersion and reflection from media formed by lattices of thin ideally conducting cylinders using the local field approach in the manner similar to that used for three-dimensional lattices of scatterers in $[15]$.

\section{FORMULATION OF THE PROBLEM}

We consider "wire media": rectangular grids of infinite wires as depicted on Figure 1. The elementary cells have dimensions $\mathbf{a} \times \mathbf{b}$. The radius of wires is $r_{0} \ll a, b$. Our main goal is to build the dispersion theory of this media and investigate the reflection of plane electromagnetic waves from a half space filled by the artificial material. It will be also very important to analyze possible descriptions of the material in terms of the effective permittivity parameter in the quasistatic limit.

Let us choose a coordinate system such that $O Z$-axis is the axis of one (reference) wire, and $O X$ and $O Y$-axes are parallel to a and $\mathbf{b}$, respectively. In this coordinate system the radius vectors of distances from the reference wire to the wire with numbers $m, n$ can be written as $\mathbf{R}_{m, n}=m \mathbf{a}+n \mathbf{b}$. We assume that the wires are thin compared to the grid periods, so that polarization perpendicular to the wire axis is very small compared to the longitudinal one. Thus, the electric field produced by the polarized wire in the volume outside the wires is equal to the electric field of current lines. In studies of eigenwaves in the infinite periodic structure we assume the plane-wave dependence 


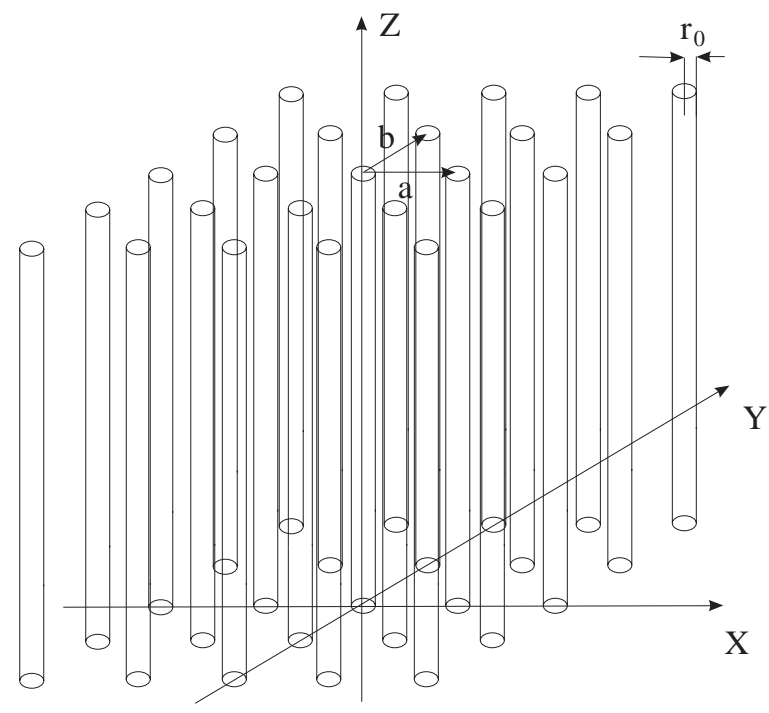

Figure 1. The geometry of wire media: an array of parallel ideally conducting thin wires.

of the current amplitudes in the form

$$
\mathbf{I}_{m, n}=\mathbf{I} e^{-j\left(q_{x} a m+q_{y} b n+q_{z} z\right)},
$$

where $\mathbf{I}$ is the current of the reference wire. The time dependance is harmonic: $e^{j \omega t}$.

The field of every wire can be described using Green's function (e.g., $[16])$

$$
G(r, z)=\frac{j}{4} H_{0}^{(2)}\left(\sqrt{k^{2}-q_{z}^{2}} r\right) e^{-j q_{z} z},
$$

where distance $r$ is measured from the wire axis, and $k$ is the free-space wave number.

We need only the expression for the longitudinal electric field component which can be obtained from (2) in the following form:

$$
\mathbf{E}(r, z)=-\frac{\eta\left(k^{2}-q_{z}^{2}\right)}{4 k} H_{0}^{(2)}\left(\sqrt{k^{2}-q_{z}^{2}} r\right) \mathbf{I} e^{-j q_{z} z},
$$

where $\eta$ is the free-space wave impedance.

The effective susceptibility of a wire excited by local electric field which depends on the coordinate along the wire like $\mathbf{E}^{\mathrm{loc}} e^{-j q_{z} z}$ can be 
found from the boundary condition on the wire surface:

$$
\begin{aligned}
\alpha & =\frac{I}{E_{z}^{\mathrm{loc}}}=\left[\frac{\eta\left(k^{2}-q_{z}^{2}\right)}{4 k} H_{0}^{(2)}\left(\sqrt{k^{2}-q_{z}^{2}} r_{0}\right)\right]^{-1} \\
& \approx\left[\frac{\eta\left(k^{2}-q_{z}^{2}\right)}{4 k}\left(1-j \frac{2}{\pi}\left\{\log \frac{\sqrt{k^{2}-q_{z}^{2}} r_{0}}{2}+\gamma\right\}\right)\right]^{-1},
\end{aligned}
$$

where $\gamma \approx 0.5772$ is the Euler constant. Thus, we can model the wires by lines of current with the known susceptibility (4).

\section{DISPERSION EQUATION}

The dispersion characteristics of the media under consideration can be found as solutions of the corresponding eigenvalue problem. We will follow deduction introduced in [15] for a three-dimensional lattice. Assuming that an eigenwave has a plane-wave spatial dependence $e^{-j\left(q_{x} x+q_{y} y+q_{z} z\right)}$, we write the expression for the local electric field acting on the reference wire:

$$
\begin{aligned}
\alpha^{-1} I & =E_{z}^{\mathrm{loc}} \\
& =-\frac{\eta\left(k^{2}-q_{z}^{2}\right)}{4 k} \sum_{(m, n) \neq(0,0)}\left[H_{0}^{(2)}\left(\sqrt{k^{2}-q_{z}^{2}} R_{m, n}\right) e^{-j\left(q_{x} a m+q_{y} b n\right)}\right] I .
\end{aligned}
$$

Using this formula we can easily write down the dispersion equation:

$$
\alpha^{-1}=C\left(k, q_{x}, q_{y}, q_{z}\right)
$$

where $C$ is the dynamic interaction constant that describes interaction effects in the infinite lattice:

$$
\begin{aligned}
C\left(k, q_{x}, q_{y}, q_{z}\right)= & -\frac{\eta\left(k^{2}-q_{z}^{2}\right)}{4 k} \\
& \sum_{(m, n) \neq(0,0)}\left[H_{0}^{(2)}\left(\sqrt{k^{2}-q_{z}^{2}} R_{m, n}\right) e^{-j\left(q_{x} a m+q_{y} b n\right)}\right] .
\end{aligned}
$$

The main difficulty there is of course calculation of the double series in the expression for the interaction constant $C$. Direct calculation of this series is not possible due to the fact that the series is diverging (in the absence of losses).

Using summation by layers corresponding to the $m$-index, we can rewrite the double series through terms $\beta(m)$ corresponding to each 
layer:

$$
\begin{gathered}
C\left(k, q_{x}, q_{y}, q_{z}\right)=\sum_{m=-\infty}^{+\infty} \beta(m) e^{-j q_{x} a m} \\
\beta(m)=-\frac{\eta\left(k^{2}-q_{z}^{2}\right)}{4 k} \sum_{n=-\infty}^{+\infty}\left[H_{0}^{(2)}\left(\sqrt{k^{2}-q_{z}^{2}} R_{m, n}\right) e^{-j q_{y} b n}\right], \text { for } m \neq 0 \\
\beta(0)=-\frac{\eta\left(k^{2}-q_{z}^{2}\right)}{4 k} \sum_{n \neq 0}\left[H_{0}^{(2)}\left(\sqrt{k^{2}-q_{z}^{2}} R_{m, n}\right) e^{-j q_{y} b n}\right]
\end{gathered}
$$

The term $\beta(0)$ corresponding to the zero-plane can be calculated using the Poisson summation formula with singularity cancellation [17]:

$$
\begin{aligned}
\beta(0)= & -\frac{\eta\left(k^{2}-q_{z}^{2}\right)}{4 k} \sum_{n \neq 0}\left[H_{0}^{(2)}\left(\sqrt{k^{2}-q_{z}^{2}} b n\right) e^{-j q_{y} b|n|}\right] \\
= & -\frac{\eta\left(k^{2}-q_{z}^{2}\right)}{2 k}\left[\frac{1}{\sqrt{k^{2}-q_{y}^{2}-q_{z}^{2}} b}-\frac{1}{2}+\frac{j}{\pi}\left(\log \frac{\sqrt{k^{2}-q_{z}^{2}} b}{4 \pi}+\gamma\right)\right. \\
& \left.+\frac{j}{b} \sum_{n \neq 0}\left(\frac{1}{\sqrt{\left(q_{y}+\frac{2 \pi n}{b}\right)^{2}+q_{z}^{2}-k^{2}}}-\frac{b}{2 \pi|n|}\right)\right] .
\end{aligned}
$$

For calculation of terms $\beta(m)$ of the other layers the Floquet representation can be applied:

$$
\begin{aligned}
\beta(m) & =-\frac{\eta\left(k^{2}-k_{z}^{2}\right)}{4 k} \sum_{n=-\infty}^{+\infty}\left[H_{0}^{(2)}\left(\sqrt{k^{2}-q_{z}^{2}} R_{m, n}\right) e^{-j q_{y} b n}\right] \\
& =-\frac{\eta\left(k^{2}-q_{z}^{2}\right)}{2 k b} \sum_{n=-\infty}^{+\infty} \frac{e^{-j k_{x}^{(n)} a|m|}}{k_{x}^{(n)}}
\end{aligned}
$$

Here $k_{x}^{(n)}$ denotes the $X$-component of $n$-th Floquet mode's wave vector:

$$
k_{x}^{(n)}=-j \sqrt{\left(q_{y}+\frac{2 \pi n}{b}\right)^{2}+q_{z}^{2}-k^{2}}, \quad \operatorname{Re}\{\sqrt{()}\}>0 .
$$


Substituting (12) into (8), changing the order of summation and using the following expression for summation of the geometrical progressions:

$$
\sum_{m=-\infty}^{\infty} e^{-j k_{x}^{(n)} a|m|-j q_{x} a m}=\frac{j \sin k_{x}^{(n)} a}{\cos k_{x}^{(n)} a-\cos q_{x} a},
$$

we obtain a very useful expression for the calculation of the dynamic interaction constant:

$$
C\left(k, q_{x}, q_{y}, q_{z}\right)=\beta(0)-\frac{\eta\left(k^{2}-q_{z}^{2}\right)}{2 k b} \sum_{n=-\infty}^{+\infty} \frac{1}{k_{x}^{(n)}}\left(\frac{j \sin k_{x}^{(n)} a}{\cos k_{x}^{(n)} a-\cos q_{x} a}-1\right)
$$

The series here has excellent convergence. Substituting (15) together with (4) and (12) into the dispersion equation (6) we get

$$
\begin{aligned}
\frac{1}{\pi} \log \frac{b}{2 \pi r_{0}} & +\frac{1}{b k_{x}^{(0)}} \frac{\sin k_{x}^{(0)} a}{\cos k_{x}^{(0)} a-\cos q_{x} a} \\
& +\sum_{n \neq 0}\left(\frac{1}{b k_{x}^{(n)}} \frac{\sin k_{x}^{(n)} a}{\cos k_{x}^{(n)} a-\cos q_{x} a}-\frac{1}{2 \pi|n|}\right)=0 .
\end{aligned}
$$

This final dispersion equation is exact for thin ideally conducting wires, the only assumption is that $r_{0} \ll a, b, \lambda$.

Let us note that we have obtained a real-valued dispersion equation. The terms corresponding to the evanescent Floquet modes are purely real:

$$
\frac{1}{b k_{x}^{(n)}} \frac{\sin k_{x}^{(n)} a}{\cos k_{x}^{(n)} a-\cos q_{x} a}=\frac{1}{b \operatorname{Im}\left\{k_{x}^{(n)}\right\}} \frac{\sinh \left(\operatorname{Im}\left\{k_{x}^{(n)}\right\} a\right)}{\cosh \left(\operatorname{Im}\left\{k_{x}^{(n)}\right\} a\right)-\cos q_{x} a}
$$

Solving the dispersion equation (16) we can obtain dependencies of the eigenwave propagation constants $q_{x}, q_{y}, q_{z}$ on the frequency $\omega$.

One can see that the dispersion curves are the lines of level corresponding to $\frac{1}{\pi} \log \frac{b}{2 \pi r_{0}}$ of the surface described by the other terms in the dispersion equation (16). This surface is plotted on Figure 2. Here, the normalized frequency is $k a /(2 \pi)=\omega \sqrt{\varepsilon_{0} \mu_{0}} a /(2 \pi)$ and $\Gamma=(0,0,0)^{T}, X=(\pi / a, 0,0)^{T}, M=(\pi / a, \pi / a, 0)$ are points in the first Brillouin zone.

We should note that the obtained dispersion equation (16) looks asymmetric with respect to an interchange of $a$ and $b$ together with $k_{x}$ and $k_{y}$. Obviously, it must be symmetric due to the symmetry 


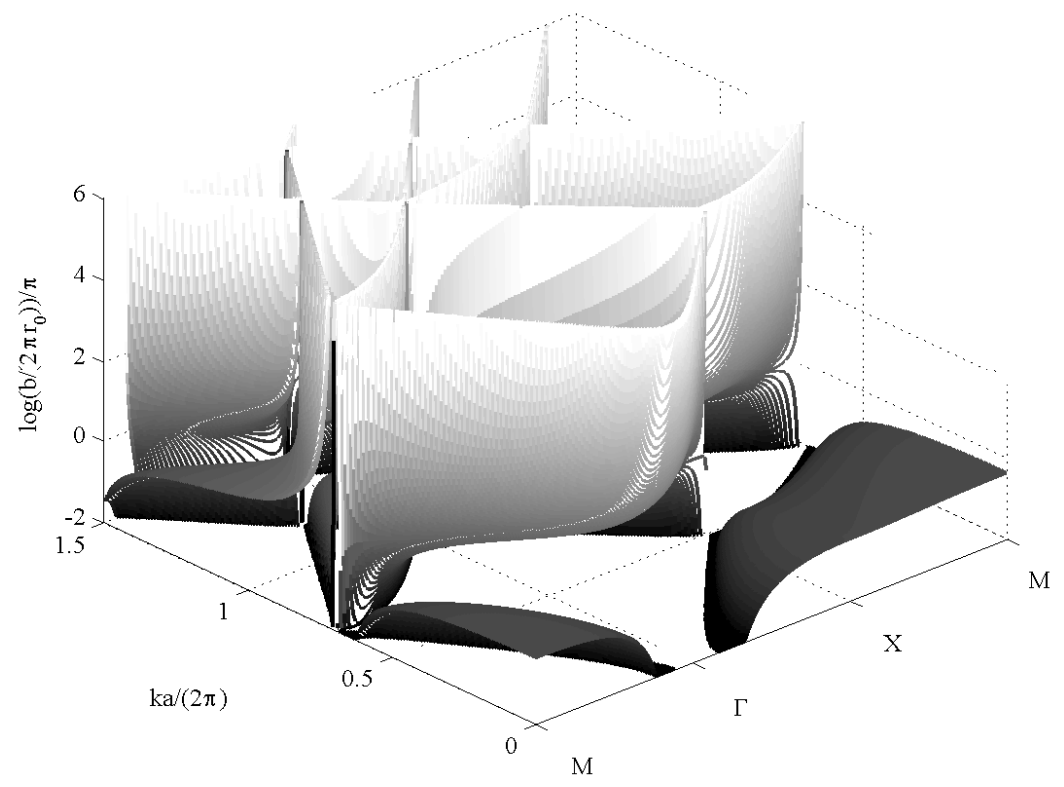

Figure 2. Illustration for the dispersion equation. The lines of level of this surface corresponding to the value $\frac{1}{\pi} \log \frac{b}{2 \pi r_{0}}$ are the dispersion curves of the eigenmodes.

of equation (7), from which we have started the derivation. We have checked numerically this fact and proved that expression (16) does possess this symmetry property. This means that one can use instead of (16) its equivalent form

$$
\begin{aligned}
& \frac{1}{\pi} \log \frac{a}{2 \pi r_{0}}+\frac{1}{a k_{y}^{(0)} \frac{\sin k_{y}^{(0)} b}{\cos k_{y}^{(0)} b-\cos q_{y} b}} \\
&+\sum_{n \neq 0}\left(\frac{1}{a k_{y}^{(n)}} \frac{\sin k_{y}^{(n)} b}{\cos k_{y}^{(n)} b-\cos q_{y} b}-\frac{1}{2 \pi|n|}\right)=0, \\
& k_{y}^{(n)}=-j \sqrt{\left(q_{x}+\frac{2 \pi n}{a}\right)^{2}+q_{z}^{2}-k^{2}, \quad \operatorname{Re}\{\sqrt{()}\}>0}
\end{aligned}
$$

with the same results.

The dispersion equation in form (16) can be easily analyzed numerically, but it is of advantage to rewrite it in the standard form adopted in the theory of periodical waveguides (e.g., $[1,18])$ :

$$
\cos q_{x} a-\cos k_{x}^{(0)} a=j \frac{\eta}{2 Z_{s}} \sin k_{x}^{(0)} a,
$$


where the effective impedance for a layer of wires reads

$$
Z_{s}=j \frac{\eta}{2}\left[\frac{b k_{x}^{(0)}}{\pi} \log \frac{b}{2 \pi r_{0}}+k_{x}^{(0)} \sum_{n \neq 0}\left(\frac{1}{k_{x}^{(n)}} \frac{\sin k_{x}^{(n)} a}{\cos k_{x}^{(n)} a-\cos q_{x} a}-\frac{b}{2 \pi|n|}\right)\right] .
$$

This impedance without taking into account the terms corresponding to the evanescent modes does not depend on the propagation constant. It means that in the plane-wave-interaction approximation (only the fundamental Floquet modes between the wire layers) the dispersion equation can be solved analytically, and we have the classical result [18]. But if all the Floquet harmonics are taken into account, the equivalent surface impedance (21) depends on the frequency and on the wavevector $Z_{s}\left(k, q_{x}, q_{y}, q_{z}\right)$, which means there are spatial dispersion effects. A similar description for arrays of small particles was suggested in [19].

Also it is instructive to write a simplified dispersion equation for the axial propagation (along $O Y$ axis, $q_{x}=q_{z}=0$ ) in the form:

$$
\begin{aligned}
& \frac{1}{\pi} \log \frac{b}{2 \pi r_{0}}=\frac{\cot \left(k_{x}^{(0)} a / 2\right)}{b k_{x}^{(0)}}+\sum_{n \neq 0}\left(\frac{\cot \left(k_{x}^{(n)} a / 2\right)}{b k_{x}^{(n)}}+\frac{1}{2 \pi|n|}\right), \\
& k_{x}^{(n)}=-j \sqrt{\left(q_{y}+\frac{2 \pi n}{b}\right)^{2}-k^{2}}, \quad \operatorname{Re}\{\sqrt{()}\}>0 .
\end{aligned}
$$

or in the equivalent symmetrically transformed form:

$$
\begin{aligned}
& \frac{1}{\pi} \log \frac{a}{2 \pi r_{0}}+\frac{1}{a k_{y}^{(0)}} \frac{\sin k_{y}^{(0)} a}{\cos k_{y}^{(0)} a-\cos q_{y} a} \\
& +\sum_{n \neq 0}\left(\frac{1}{a k_{y}^{(n)}} \frac{\sin k_{y}^{(n)} b}{\cos k_{y}^{(n)} b-\cos q_{y} b}-\frac{1}{2 \pi|n|}\right)=0, \\
& k_{y}^{(n)}=-j \sqrt{\left(\frac{2 \pi n}{a}\right)^{2}-k^{2}}, \quad \operatorname{Re}\{\sqrt{()}\}>0 .
\end{aligned}
$$

In $(22)$ the dependence on $q_{y}$ is only through parameters $k_{x}^{(n)}$, but in $(22)$ the values $k_{y}^{(n)}$ are independent on $q_{y}$. Thus, we have a different in form but equivalent expressions (22) and (24) of the dispersion equation for the axial propagation. One can use any of these formulae with the same result.

To verify the introduced theory, a comparison with the results obtained in [14] has been done. The dispersion curves for a square 
grid of cylinders with the filling ratio $f=\pi r_{0}^{2} / a^{2}=0.001$ have been calculated using the introduced technique, and the result is shown on Figure 3, where $\Gamma=(0,0,0)^{T}, X=(\pi / a, 0,0)^{T}$, and $M=(\pi / a, \pi / a, 0)$ are points in the first Brillouin zone. Here, together with the thick lines representing the dispersion curves for the wire medium, the dispersion curves for free space are presented for comparison (as thin lines). Excellent correspondence between our result and results from [4] and [14] is observed.

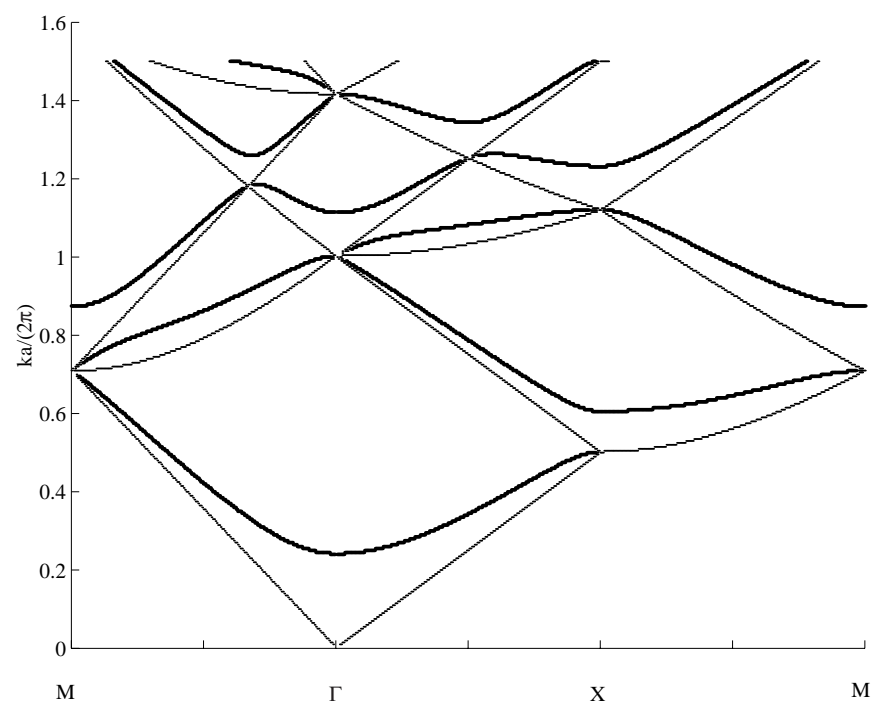

Figure 3. Dispersion curves for a square grid of cylinders with the filling ratio $f=0.001$ (thick lines) and dispersion curves for free space (thin lines).

\section{REFLECTION COEFFICIENT}

Let us consider a plane interface between a half space filled with a wire medium $(x>0$ or index $m \geq 0)$ and free space. Suppose that an incident plane electromagnetic wave $\mathbf{E} e^{-j\left(k_{x} x+k_{y} y+k_{z} z\right)}$ is exciting the medium. If we neglect the transition layer and assume that even the first raw of wires is excited in the same way as wires in the bulk, the layer-to-layer distribution of the currents will be given by (1), where $q_{x}$ is the propagation constant which can be found as a solution of dispersion equation (16) with $q_{y}=k_{y}, q_{z}=k_{z}$. This also means that we consider the situation when there is only one propagating mode in 
the medium. In the theory of dielectrics it is known that the surface correction is actually rather small [15]. Let us write the equations for the current in some $M$-th layer located deeply inside the medium $(M \gg 1)$ through the local electric field produced by the wires and the incident wave:

$$
I e^{-j q_{x} a M}=\alpha\left(E_{z} e^{-j k_{x} a M}+\left[\sum_{m=-M}^{+\infty} \beta(m) e^{-j q_{x} a m}\right] I e^{-j q_{x} a M}\right) .
$$

Direct calculation of the series in the right side of this equation is a complicated problem, but we can take into account the fact that the layer-to-layer distribution is described by the propagation constant which is a solution of the dispersion equation for the corresponding infinite medium. Using formulae (6) and (8) together we have

$$
\sum_{m=-\infty}^{+\infty} \beta(m) e^{-j q_{x} a m}=\alpha^{-1}
$$

which leads to

$$
\sum_{m=-M}^{+\infty} \beta(m) e^{-j q_{x} a m}=\alpha^{-1}-\sum_{m=-\infty}^{-M-1} \beta(m) e^{-j q_{x} a m} .
$$

For layers with large numbers $m$ the evanescent Floquet modes in expression (12) for interaction parameters $\beta(m)$ can be neglected:

$$
\beta(-m) \approx-\frac{\eta\left(k^{2}-q_{z}^{2}\right)}{2 k b} \frac{e^{-j k_{x} a m}}{k_{x}}, \quad m \gg 1 .
$$

Thus,

$$
\sum_{m=-\infty}^{-M-1} \beta(m) e^{-j q_{x} a m}=-\frac{\eta\left(k^{2}-q_{z}^{2}\right)}{2 k b k_{x}} \frac{e^{-j\left(k_{x}-q_{x}\right) a(M+1)}}{1-e^{-j\left(k_{x}-q_{x}\right) a}} .
$$

Further, we get

$$
I=-\left[\frac{\eta\left(k^{2}-q_{z}^{2}\right)}{2 k b k_{x}} \frac{e^{-j\left(k_{x}-q_{x}\right) a}}{1-e^{-j\left(k_{x}-q_{x}\right) a}}\right]^{-1} E_{z} .
$$

The reflected electric field produced by the excited lattice is a sum of plane waves generated by all the layers:

$$
\begin{aligned}
E_{R} & =-\frac{\eta\left(k^{2}-q_{z}^{2}\right)}{2 k b k_{x}} \sum_{m=0}^{+\infty} e^{-j\left(k_{x}+q_{x}\right) a m} I \\
& =-\frac{\eta\left(k^{2}-q_{z}^{2}\right)}{2 k b k_{x}} \frac{I}{1-e^{-j\left(k_{x}+q_{x}\right) a}} .
\end{aligned}
$$


Finally, the reflection coefficient reads

$$
R=-\frac{1-e^{-j\left(q_{x}-k_{x}\right) a}}{1-e^{-j\left(q_{x}+k_{x}\right) a}}=e^{j k_{x} a} \frac{\sin \left[\left(k_{x}-q_{x}\right) a / 2\right]}{\sin \left[\left(k_{x}+q_{x}\right) a / 2\right]} .
$$

The result depends on the reference plane. It appears of advantage to rewrite the above equation for the plane at a half-period distance from the first wire, measured inward the medium:

$$
R=\frac{\sin \left[\left(k_{x}-q_{x}\right) a / 2\right]}{\sin \left[\left(k_{x}+q_{x}\right) a / 2\right]} .
$$

This value is real for propagating modes ( $q_{x}$ is purely real). For decaying modes inside a stop band (when $q_{x}$ is purely imaginary) we have full power reflection: $|R|=1$.

Also we should note, that we have deducted (34) for modes propagating from free space into the half space filled by wires. It means that we should take the correct sign of $q_{x}$ to insert it in these formulae in accordance with the dispersion sign. We solve the dispersion equation for real propagation constants $\tilde{q}_{x}$ in the range $[0 \ldots \pi / a]$, and for the single-mode regime $k a /(2 \pi)<1$ we should take $q_{x}=\tilde{q}_{x}$, if $k a /(2 \pi)<0.5$, but $q_{x}=-\tilde{q}_{x}$, if $k a /(2 \pi)>0.5$. From the two solutions having a nonzero imaginary part of the propagation constant we should choose decaying ones.

As a numerical example we have calculated the reflection coefficient from a half-space filled by a square grid of cylinders with the filling ratio $f=0.001$ in the single-mode regime $(k a<2 \pi)$. The result is shown in Figure 4 as a function of the normalized frequency $k a /(2 \pi)$. The reflection coefficient equals to -1 at very low frequencies. Within the low-frequency stop band it changes its phase, so that at the top edge of the band gap at $k a /(2 \pi) \approx 0.25$ the reflection coefficient becomes equal to +1 . In other words, the interface behaves as a metal wall at $k a \rightarrow 0$, but it reflects as a magnetic wall at frequencies close to the first propagation window. The reflection coefficient remains positive in the first propagation region $0.25<k a /(2 \pi)<0.5$, and it again becomes equal to +1 at the bottom edge $k a=\pi$ of the second stop band, and after that it changes phase inside the stop band at frequencies $k a=\pi$ to $k a /(2 \pi) \approx 0.6$, and becomes equal to -1 at the top edge. In the second propagation window from $k a /(2 \pi) \approx 0.6$ to $k a=2 \pi$ the reflection coefficient is negative.

\section{DENSE GRID}

For dense compared with the wavelength grids the dispersion equation (16) can be simplified using the Taylor expansion of sin and 

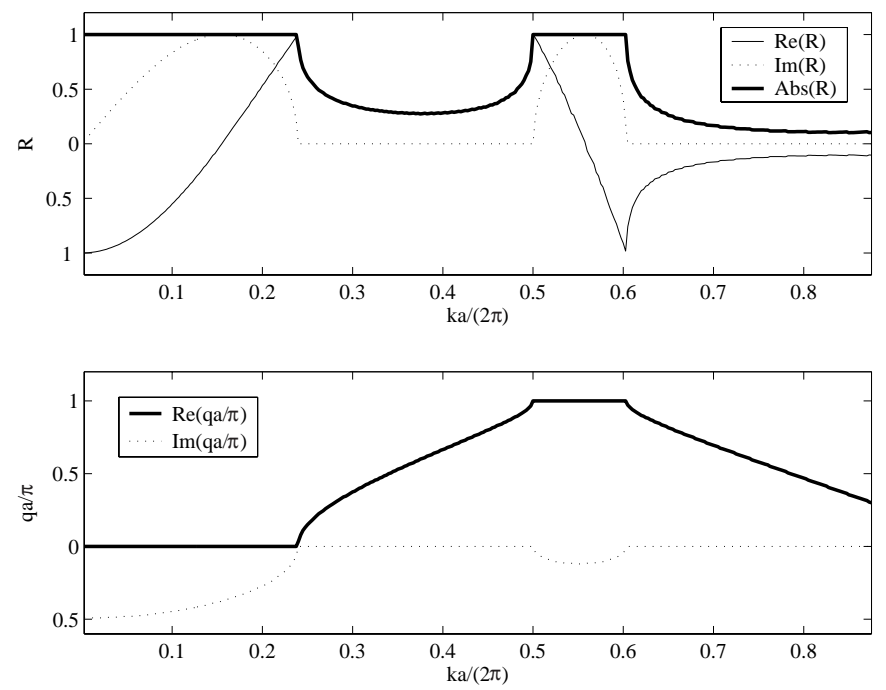

Figure 4. Dependence of the reflection coefficient from a half space filled by a square grid of cylinders with the filling ratio $f=0.001$ and the corresponding propagation constant on the normalized frequency.

cos functions of small arguments and analytically solved. The result is the following:

$$
q^{2}=q_{x}^{2}+q_{y}^{2}+q_{z}^{2}=k^{2}-k_{0}^{2},
$$

where

$$
k_{0}^{2}=\frac{2 \pi /(a b)}{\log \frac{b}{2 \pi r_{0}}+\sum_{n=1}^{+\infty}\left(\frac{\operatorname{coth} \frac{\pi n a}{b}-1}{n}\right)+\frac{\pi a}{6 b}} .
$$

Thus, there is a stop band at low frequencies with the upper boundary at the frequency corresponding to $k_{0}$ : for $k<k_{0} q=$ $-j \sqrt{k_{0}^{2}-k^{2}}$, and for $k>k_{0} q=\sqrt{k^{2}-k_{0}^{2}}$. It is interesting to observe that $k_{0}$ as a function of the lattice constants $a$ and $b$ is symmetric: $k_{0}(a, b)=k_{0}(b, a)$. This follows from the described above symmetry property of the dispersion equation (16), and also can be easily numerically checked. This fact is physically sound an obvious, but it is not so easy to see it from expression (36).

If we introduce instead of the lattice parameters $a$ and $b$ their 


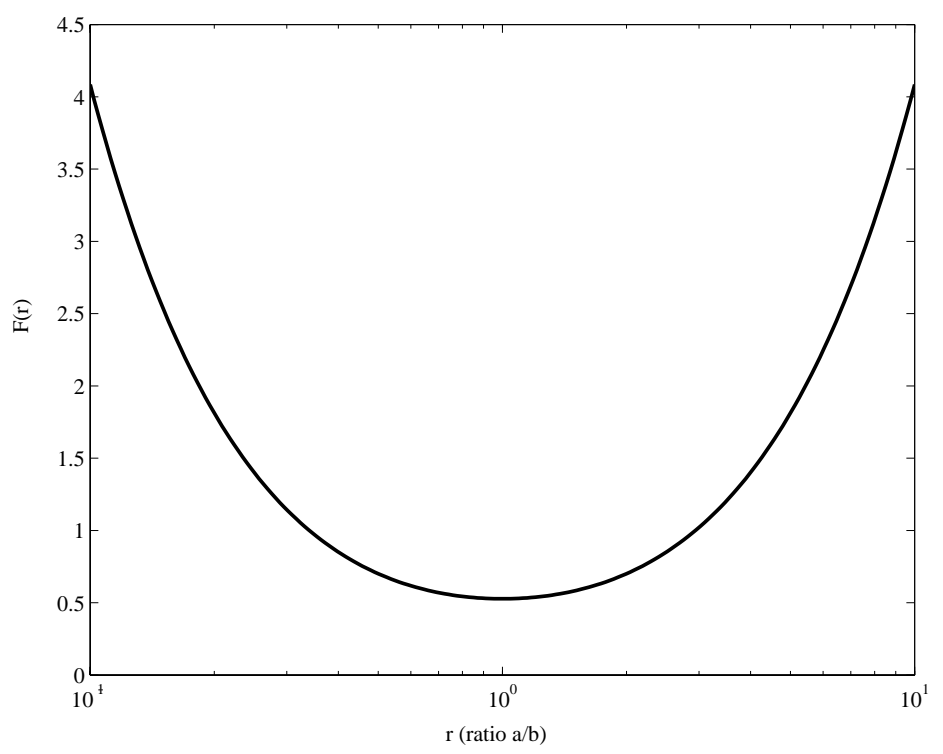

Figure 5. Plot of function $F(r)$.

product $s=\sqrt{a b}$ and their ratio $r=a / b$, we can rewrite (36) as

$$
k_{0}^{2}=\frac{2 \pi / s^{2}}{\log \frac{s}{2 \pi r_{0}}+F(r)},
$$

where

$$
F(r)=-\frac{1}{2} \log r+\sum_{n=1}^{+\infty}\left(\frac{\operatorname{coth}(\pi n r)-1}{n}\right)+\frac{\pi r}{6},
$$

The plot of function $F(r)$ is presented on Figure 5 . We can see that the value of $k_{0}$ is maximal at $r=1$ for a fixed cell area. A numerical investigation shows that function $F(r)$ can be approximated in the following manner:

$$
F(r) \approx \frac{\pi}{6}(r+1 / r)-\sqrt{\log ^{2}(r)+(\pi / 3)^{2}} / 2 .
$$

Such an approximation is exact for $r \rightarrow+\infty$ and $r \rightarrow 0$ respectively, but for other parameters it gives a small error (maximum 3.5 percent). This approximation is adopted to give the best result for $r=1$, where $F(1)=0.5275 \approx \pi / 6$. 
For the square grid $(a=b)$ expression (36) simplifies, and we have

$$
k_{0}^{2}=\frac{2 \pi / a^{2}}{\log \frac{a}{2 \pi r_{0}}+0.5275} .
$$

The normalized width of the stop band as a function of the ratio $a / r_{0}$ is plotted on Figure 6, where we compare the exact result obtained from a numerical solution of (16) and the approximate formula (40). The correct numbers correspond to the part of the plot outside the filled rectangle $r<0.1 a$, due to the restrictions of our theory to wires with small radii.

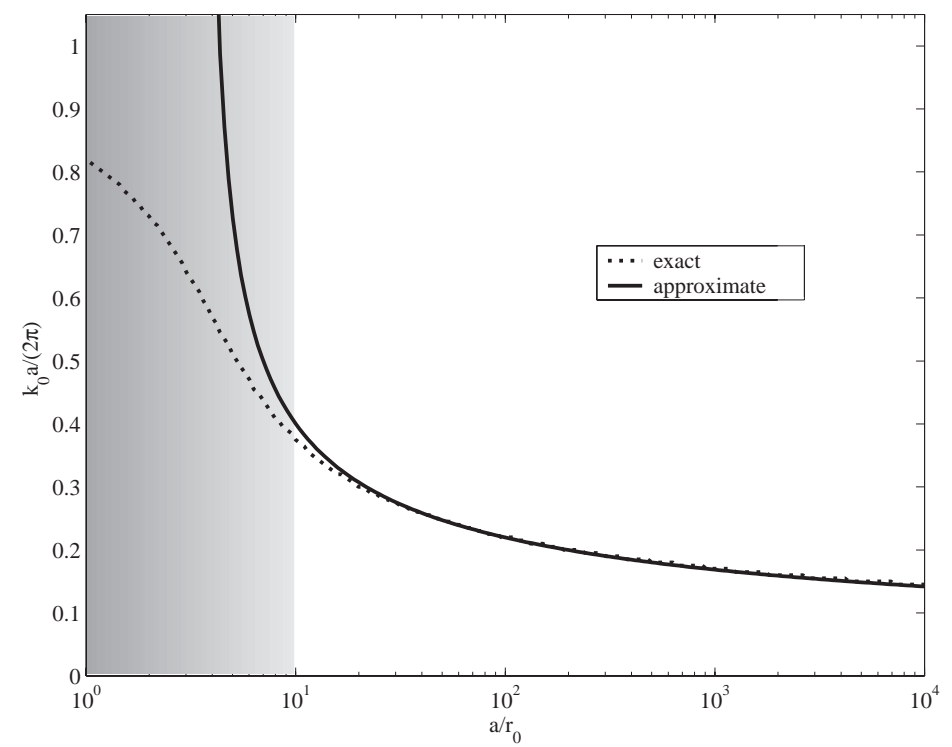

Figure 6. Normalized width of the stop band as a function of ratio $a / r_{0}$ for square grids

The media under consideration in the quasistatic limit do not possess any magnetic properties, and the dispersion equation (35) can be reformulated in terms of a frequency dependent permittivity:

$$
\varepsilon_{\mathrm{eff}}=\varepsilon_{0}\left(1-\frac{k_{0}^{2}}{k^{2}}\right) .
$$

This is a well-known classical result [13], which shows that $\varepsilon_{\text {eff }}<0$ for $k<k_{0}$ and $0<\varepsilon_{\text {eff }}<1$ for $k>k_{0}$. 


\section{CONCLUSION}

We have presented an analytical theory of dispersion and reflection from media formed by rectangular lattices of thin ideally conducting cylinders using the local field approach. The transcendental dispersion equation has been obtained in closed form and solved numerically. The dispersion curves and the reflection coefficient for a square grid of cylinders with the filling ratio $f=\pi r_{0}^{2} / a^{2}=0.001$ have been calculated using the introduced technique, and results are shown on Figures 3 and 4, respectively. The results have been successfully verified by comparison with the dispersion curves presented in [4] and [14]. It has been shown that wire media can be described by a negative permittivity in the low frequency regime, and a simple analytical formula for the frequency dependent effective permittivity in the plasmon-like form is presented. In contrast to the classical analysis [1], this formula takes into account higher-order Floquet mode interactions in the lattice. The more recent analysis of wire media [3] requires intensive numerical calculations for an estimation of the effective permittivity even at low frequencies.

\section{REFERENCES}

1. Brown, J., "Artificial dielectrics," Progress in Dielectrics, Vol. 2, 195-225, 1960.

2. King, R. J., D. V. Thiel, and K. S. Park, "The synthesis of surface reactance using an artificial dielectric," IEEE Trans. Antennas and Propagat., Vol. 31, No. 3, 471-476, 1983.

3. Moses, C. A. and N. Engheta, "Electromagnetic wave propagation in the wire medium: a complex medium with long thin inclusions," Wave Motion, Vol. 34, 301-317, 2001.

4. Nicorovichi, N. A., R. C. McPhedran, and L. C. Botten, "Photonic band gaps for arrays of perfectly conducting cylinders," Physical Review E, Vol. 52, No. 1, 1135-1145, 1995.

5. Chin, S. K., N. A. Nicorovici, and R. C. McPhedran, "Green's function and lattice sums for electromagnetic scattering by a square array of cylinders," Physical Review E, Vol. 49, No. 5, 4590-4602, 1994.

6. Kuzmiak, V., A. A. Maradudin, and F. Pincemin, "Photonic band structures of two-dimensional systems containing metallic components," Physical Review B, Vol. 50, No. 23, 16835-16844, 1994.

7. Kuzmiak, V., A. A. Maradudin, and A. R. McGurn, "Photonic 
band structures of two-dimensional systems fabricated from rods of a cubic polar crystal," Physical Review B, Vol. 55, No. 7, 42984311, 1997.

8. Sakoda, K., N. Kawai, T. Ito, A. Chutinan, S. Noda, T. Mitsuyu, and K. Hirao, "Photonic bands of metallic systems. I. Principle of calculation and accuracy," Physical Review B, Vol. 64, 045116, 2001.

9. Ito, T. and K.Sakoda, Photonic bands of metallic systems. II. Features of surface plasmon polaritons, Physical Review B, Vol. 64, 045117, 2001.

10. Sigalas, M. M., C. T. Chan, K. M. Ho, and C. M. Soukoulis, "Metallic photonic band-gap materials," Physical Review B, Vol. 52, No. 16, 11744-11751, 1995.

11. Sigalas, M., C. M. Soukoulis, E. N. Economou, C. T. Chan, and K. M. Ho, "Photonic band gaps and defects in two dimensions: Studies of the transmission coefficient," Physical Review B, Vol. 48, No. 19, 14121-14126, 1993.

12. Pendry, J. B. and A. MacKinnon, "Calculation of photon dispersion relations," Physical Review Letters, Vol. 69, No. 19, 2772-2775, 1992.

13. Guida, G., D. Maystre, G. Tayeb, and P. Vincent, "Mean-field theory of two-dimensional metallic photonic crystals," J. Opt. Soc. Am. B, Vol. 15, No. 8, 2308-2315, 1998.

14. Simovski, C. R., M. Qiu, and S. He, "Averaged field approach for obtaining the band structure of a photonic crystal with conducting inclusions," J. Electromagn. Waves Applic., Vol. 14, 449-468, 2000.

15. Mahan, G. D. and G. Obermair, "Polaritons at surfaces," Physical Review, Vol. 183, No. 3, 834-841, 1969.

16. Felsen, L. B. and N. Marcuvitz, Radiation and Scattering of Waves, Prentice-Hall, Englewood Cliffs, NJ, 1972.

17. Collin, R. E., Field Theory of Guided Waves, 2nd Edition, IEEE Press, Piscataway, NJ, USA, 1990.

18. Collin, R. E., Foundations for Microwave Engineering, McGrawHill, New York, 1966.

19. Tretyakov, S. A. and A. J. Viitanen, "Plane waves in regular arrays of dipole scatterers and effective-medium modeling," J. Opt. Soc. Am. A, Vol. 17, No. 10, 1791-1796, 2000.

20. Yatsenko, V. V., S. A. Tretyakov, S. I. Maslovski, and A. A. Sochava, "Higher order impedance boundary conditions for sparse wire grids," IEEE Trans. Antennas Propag., Vol. 48, No. 5, 720-727, 2000. 
21. Pendry, J. B., A. J. Holden, W. J. Stewart, and I. Youngs, "Extremety low frequency plasmons in metallic mesostructures," Phys. Rev. Lett., Vol. 76, No. 25, 4773-4776, 1996.

Pavel A. Belov received the B.Sc. and M.Sc. in mathematics from the St. Petersburg Institute of Fine Mechanic and Optics (Russia) in 1998 and 2000, respectively. Since 2000 he has been Ph.D. student of the Physics Department of the St. Petersburg Institute of Fine Mechanic and Optics. Since 2001 he has been researcher and Ph.D. student in the Radio Laboratory, Helsinki University of Technology (Finland). He received 1999 and 2000 SPIE Scolarships, 2001 IEEE MTT-S Fellowship, and 2001 INTAS Fellowship. His scientific interests include analytical and numerical modeling of electromagnetic properties of photonic crystals, artificial microwave periodic structures, composite materials and high impedance surfaces.

Sergei A. Tretyakov received the Dipl. Engineer-Physicist, the Ph.D., and the Doctor of Sciences degrees (all in radiophysics) from the St. Petersburg State Technical University (Russia), in 1980, 1987, and 1995, respectively. From 1980 to 2000 he was with the Radiophysics Department of the St. Petersburg State Technical University. Presently, he is professor of radio engineering in the Radio Laboratory, Helsinki University of Technology. His main scientific interests are electromagnetic field theory, complex media electromagnetics and microwave engineering. Prof. Tretyakov served as Chairman of the St. Petersburg IEEE ED/MTT/AP Chapter from 1995 to 1998.

Ari J. Viitanen received the Dipl. Eng., Lic. Tech., and Dr. Tech. degrees in electrical engineering from the Helsinki University of Technology, Finland in 1984, 1989, and 1991, respectively. From 1985 to 1989 he was a Research Engineer with the Nokia Research Center. Since 1990 he has been with the Electromagnetics Laboratory, Helsinki University of Technology. His main interests are electromagnetic field theory, complex media and microwave engineering. 